\title{
Correlation Studies Between Big Five Personality Traits, Age and Loneliness
}

\author{
Neha Dalal ${ }^{1}$, Ankita Kathad ${ }^{2}$, Sameer Masurkar ${ }^{3}$, Shreya Pavithran $^{4}$, Tanisha Soumyanarayan $^{5}$ \\ ${ }^{1}$ Assistant Professor, ${ }^{2-5}$ TYBA students \\ Department of Psychology, L.S Raheja College of Arts and Commerce, Mumbai. \\ Corresponding author: Ms. Neha Dalal \\ Email-neha.dalal@1sraheja.org
}

\begin{abstract}
Background: This study investigates variables of age; openness to experiences, agreeableness, conscientiousness, extraversion and neuroticism explained by McCrae and Costa in Big Five theory; and its correlation with loneliness.

Methods: Through method of convenience sampling, 15 participants were chosen between age group of 19 to 25 years and 15 participants in the age group 40 to 50 years. Big Five Inventory (John, O.P., Srivastava, S.; 1999) and Revised UCLA Loneliness scale, $3^{\text {rd }}$ version (Russell, D., Peplau, L.A., Cutrona, C.E.; 1980) were used to collect personality dimension scores and loneliness score. Statistical procedure of multiple regression and correlation was used.

Results: Results displayed a positive correlation between neuroticism and loneliness for both age groups 19 to 25 years and 40 to 50 years. However, there was a negative correlation between conscientiousness and loneliness for age group 19 to 25 years; and negative correlation between extraversion and loneliness for age group 40 to 50 years.

Conclusion: It can be concluded that there is a significant relationship between certain personality traits and loneliness for specific age groups.
\end{abstract}

Keywords: Openness to experience, Conscientiousness, Extraversion, Agreeableness, Neuroticism, Loneliness.

(Paper received $-2^{\text {nd }}$ January 2018, Peer review completed $-8^{\text {th }}$ January 2018)

(Accepted $-12^{\text {th }}$ January 2018)

\section{INTRODUCTION}

Loneliness - Loneliness is an emotional response to isolation. It is usually complex and unpleasant. It involves anxious feelings and a lack of connection with others. Studies have documented that loneliness is an unpleasant and widespread experience [1]. Loneliness is associated with a variety of social problems like juvenile delinquency, alcohol abuse and suicide [1]. There are a few possible behavioural causes of loneliness according to Perlman and Peplau. The extent to which loneliness creates anxiety determines the level of behavioural characteristics of these states exhibited by lonely individuals. Studies show that loneliness is correlated to lack of assertiveness [1]. People who are found to be lonely seem to have an issue with voicing it to others.

In 2004, NSSO of India indicated that 1.23 million men and 3.68 million women were living alone and faced loneliness. In 2017, Age Well foundation conducted a survey titled 'Changing Needs and Rights of Older People in India'. It was presented across 300 districts of 25 states and Union Territories of India and a sample size of 15,000 older people was used. The survey indicated that 47.49 per cent elderly people in India suffer from loneliness. 


\section{Statement of problem}

The number of people who are lonely or suffer from loneliness has become increasingly common and has long term sustenance. There may be many factors which are linked to loneliness. Some of the factors impacting loneliness are gender [2], health [3], social isolation [4], divorce [5].

The researchers here were interested in investigating factors that are more internal, impacting loneliness like personality traits of individuals.

For this reason, the Big five factors of personality which is one of the most popular theories of personality, were measured and correlated to loneliness. Age was considered as the mediating factor. The researchers were also interested to see if personality variables as given in the theory could also be utilized to predict loneliness.

The Five factor model given by Costa and McCrae applies factor analysis to personality survey data.

The five factors are:

1. Openness to experience - Words like artistic, curious, imaginative, insightful and original are used to describe such people [6]. They are people who appreciate art, emotion, adventure, unusual ideas and variety of experiences. These people are intellectually high, curious, creative, they prefer novelty, and like to act spontaneously and dislike a strict routine.

2. Conscientiousness - Adjectives like efficient, organized, plan-ful, reliable, responsible and thorough are used to describe such people [6]. People who are high on conscientiousness are organised and dependable. They prefer to act out through well prepared plans over spontaneity.

3. Extraversion-Describes the extent to which people are outgoing or shy. It includes such traits as being talkative, sociable, adventurous and eager to be in the limelight $[6,7]$. People high on extraversion seek stimulation in the company of others.

4. Agreeableness -Describes the extent to which people are good natured, cooperative and secure. It reflects the tendency to have friendly relationships [7]. Agreeable people tend to be compassionate. They are believed to be naive and submissive.

5. Neuroticism -Describes the extent to which a person suffers from such traits as anxiety, an inability to control impulses and a tendency to feel negative emotions such as anger, guilt, contempt and resentment. Neurotic individuals are worriers, complainers and defeatists even when they have no major problem. They are always ready to see the sour side of life and none of its sweetness [7].

The oldest publication about loneliness is Uber die Einsamkeit [8]

They defined loneliness as "the unpleasant experience that occurs when a person's network of social relations is deficient in some important way, either quantitatively or qualitatively" [1].

Social isolation concerns the objective characteristics of a situation and refers to absence of relationships with other people [1]. Personality and interpersonal predictors of loneliness in two cultures [9]. 259 mainland US undergraduates and 332 Puerto Rican undergraduates were used for two reasons. (a) To assess cross-cultural variability in the correlations of loneliness that was previously reported. (b) To find the relative contributions of such predictors to the experience of loneliness for each sample.

There was reportedly greater loneliness in the Puerto Rican sample; according to the results. There were differences in most personality and interpersonal variables between the groups. However, the correlations of loneliness were quite similar for both samples. Factor analyses of the correlations of loneliness revealed interpretable factors that preserved the ability of the original variables to predict loneliness.

The data was consistent with the hypothesis that culture does influence the specific values observed for personality and related measures, whereas the fundamental organisation of personality remains stable across cultures. Cultural factors are considered as possible explanations for the few group differences in the predictors of loneliness.

Loneliness and divorce were seen to be associated. The greatest feelings of loneliness were in social situations when women felt like they had nobody to share responsibility; when they felt that finances were a limiting factor. When they felt out of place at a certain time or event, both men and women were found to be lonely [10-11]. 


\section{METHODOLOGY}

An observational survey design was used.The variables here were the Big Five personality traits (openness to experience, conscientiousness, extraversion, agreeableness, neuroticism) and age groups of 19-25 years and $40-50$ years.

Another variable was the score on the Loneliness scale which is designed to measure one's subjective feelings of loneliness as well as feelings of social isolation.

The purpose was to find correlation between these variables and predict significance of variables for loneliness.

The hypothesis of this research is that the Big Five Factors (openness to experience, conscientiousness, extraversion, agreeableness, neuroticism) are differentially correlated to loneliness for the age group of 19 to 25 years. Additionally, the Big Five Factors (openness to experience, conscientiousness, extraversion, agreeableness, neuroticism) are differentially correlated to loneliness for the age group of 40 to 50 years.

The sample consisted of age groups of 19 to 25 years and 40 to 50 years. A convenient sample was used wherein both the Personality and Loneliness scales were administered on students in the researchers' class itself and their respective parents.

\section{Tools used}

Tools used were the two scales:

1. The Big Five Inventory (BFI) [12]

This scale as a Likert - type rating scale with responses ranging from 'Disagree strongly' with score 1 up to 'Agree strongly' with score 5.

Items $5,10,15,20,25,30,35,40,41$ and 44 measured openness to experience. Items $3,8,13,18,23,28$, 33,38 and 43 measured conscientiousness. Items 1, 6, 11, 16, 21, 26, 31 and 36 measured extraversion. Items 2, 7, 12, 17, 22, 27, 32, 37 and 42 measured agreeableness. Items 4, 9, 14, 19, 24, 29, 34 and 39 measure neuroticism.

However, items 6, 21, 31, 2, 12, 27, 37, 8, 18, 23, 43, 9, 24, 34, 35 and 41 were reverse scored items. A meta-analysis was done to gather reliabilities of personality scale score. The dimension of personality being rated does not appear to strongly moderate the internal consistency or the test retest reliabilities.

2. Revised UCLA Loneliness scale [13]

This scale had a Likert-type rating scale with responses on this scale ranging from 'never' which is scored 1 up to 'often' with the score 4.

Items 1, 5, 6, 9, 10, 15, 16, 19, 20 were reverse scored items.

Two studies were designed to counter the effect of bias in the original scale. There are reports of concurrent validity evidence. Loneliness is considered to be distinct psychological experience [13].

\section{RESULTS}

Table 1 - Correlation Matrix between OCEAN and loneliness for 19 to 25 years

\begin{tabular}{|c|c|c|c|c|c|c|}
\hline & $\mathbf{O}$ & $\mathbf{C}$ & $\mathbf{E}$ & $\mathbf{A}$ & $\mathbf{N}$ & $\mathbf{L}$ \\
\hline $\mathbf{O}$ & 1 & 0.269 & 0.221 & 0.229 & -0.309 & -0.581 \\
\hline $\mathbf{C}$ & 0.269 & 1 & 0.347 & 0.105 & -0.16 & -0.669 \\
\hline $\mathbf{E}$ & 0.221 & 0.347 & 1 & -0.016 & -0.821 & -0.406 \\
\hline $\mathbf{A}$ & 0.229 & 0.105 & -0.016 & 1 & -0.077 & -0.437 \\
\hline $\mathbf{N}$ & -0.309 & -0.16 & -0.821 & -0.077 & 1 & 0.329 \\
\hline $\mathbf{L}$ & -0.581 & -0.669 & -0.406 & -0.437 & 0.329 & 1 \\
\hline
\end{tabular}


Table 2 - Correlation matrix between OCEAN and Loneliness for 40 to 50 years

\begin{tabular}{|c|c|c|c|c|c|c|}
\hline & $\mathbf{O}$ & $\mathbf{C}$ & $\mathbf{E}$ & $\mathbf{A}$ & $\mathbf{N}$ & $\mathbf{L}$ \\
\hline $\mathbf{O}$ & 1 & 0.476 & 0.473 & 0.281 & -0.245 & -0.311 \\
\hline $\mathbf{C}$ & 0.476 & 1 & 0.008 & 0.19 & 0.027 & 0.156 \\
\hline $\mathbf{E}$ & 0.473 & 0.008 & 1 & 0.043 & -0.386 & -0.385 \\
\hline $\mathbf{A}$ & 0.281 & 0.19 & 0.043 & 1 & -0.048 & -0.144 \\
\hline $\mathbf{N}$ & -0.245 & 0.027 & -0.386 & -0.048 & 1 & 0.825 \\
\hline $\mathbf{L}$ & -0.311 & 0.156 & -0.385 & -0.144 & 0.825 & 1 \\
\hline
\end{tabular}

Figure 1 - Correlation matrix between OCEAN and Lonliness for ages 19-25 and 40-50.

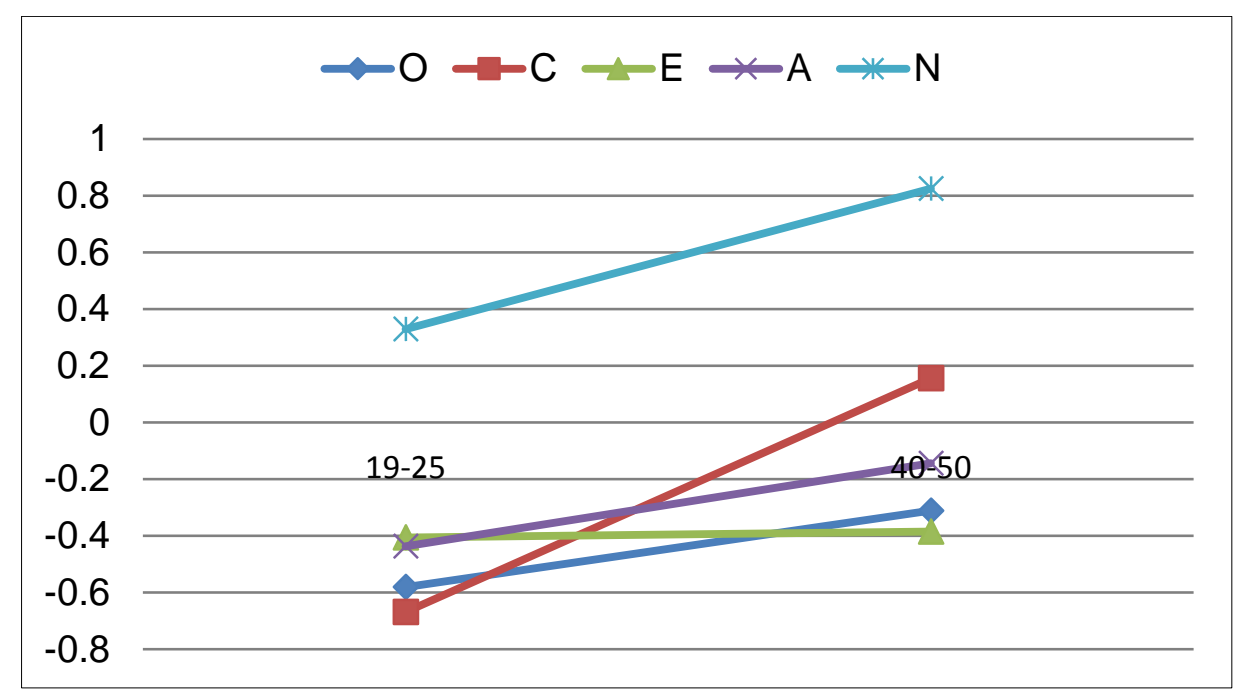

According to Table 1: Correlation matrix between OCEAN and Loneliness for 19 to 25 years and Figure 1: Correlation Matrix between OCEAN and Loneliness for ages 19 to 25 years and 40 to 50 years; there is a moderate negative correlation between openness to experiences and loneliness with the value -0.581 .

There is a high negative correlation between conscientiousness and loneliness with -0.669 . There is a moderate negative correlation between extraversion and loneliness with the value of -0.406 . There is a moderate negative correlation between agreeableness and loneliness with -0.437 as the value. There is a positive correlation between neuroticism and loneliness with the value 0.329 . As can be seen in Table 2: Correlation Matrix between OCEAN and Loneliness for 40 to 50 years and Figure 1: Correlation Matrix between OCEAN and Loneliness for ages 19 to 25 years and 40 to 50 years, the correlation between openness and loneliness is -0.311 ; between conscientiousness and loneliness is 0.156 ; between extraversion and loneliness is -0.385 ; between agreeableness and loneliness is -0.144 ; between neuroticism and loneliness is 0.825 . Therefore, it can be observed that there is a high positive correlation between neuroticism and loneliness.

\section{DISCUSSION}

According to Table 1: Correlation Matrix between OCEAN and Loneliness for 19 to 25 years and Figure 1: Correlation Matrix between OCEAN and Loneliness for ages 19 to 25 years and 40 to 50 years, there is a moderate negative correlation between openness to experiences and loneliness; extraversion and loneliness; and agreeableness and loneliness. This indicates that if individual scores high on any of these personality traits, he will score low in the scale of loneliness, i.e. the individual is less likely to experience episodes of loneliness. 
There is a positive correlation between neuroticism and loneliness. This indicates that individuals who score high on the neurotic personality trait are more likely to experience loneliness.

Neuroticism can be distinguished by the anxiety and emotional instability it brings in an individual. Individuals in their early adulthood often go through identity crises. They go through 'youth', a period of great change. Thus, for the age group of 19 to 25 years, neuroticism can be a significant predictor of loneliness.

People with a neurotic or anxious personality might harbour unrealistic relationship standards, and their unmet social needs might give rise to feelings of loneliness [14]. People with poor social skills and psychological resources are likely to experience difficulty developing and maintaining relation- ships, and for that reason might feel lonely [15].

In Table 2: Correlation Matrix between OCEAN and Loneliness for 40 to 50 years and Figure 1: Correlation Matrix between OCEAN and Loneliness for ages 19 to 25 years and 40 to 50 years, correlation between neuroticism and loneliness and conscientiousness and loneliness is positive, that is, people who score high on neuroticism and conscientiousness are more likely to experience loneliness as well.

There is a negative correlation between openness to experience and loneliness, agreeableness and loneliness and extraversion and loneliness, that is, an individual scoring high on these traits is less likely to experience loneliness.

Conscientiousness and agreeableness are positive predictors of loneliness, that is, their presence in a person can predict loneliness. Since this trait signifies how disciplined, organized, compassionate and generous one is, it increases with age as people mature and give importance to family life.

This goes to show why these two personality traits cannot be seen in people in the age group 19 to 25 years as such individuals are still 'growing up'. This also explains why neuroticism as a personality trait cannot be seen in people in the age group 40 to 50 years, since such individuals are likely to be more settled in life. Extraversion is a negative predictor of loneliness in the age group 40 to 50 years, that is, the absence of extraversion predicts the presence of loneliness. Characteristics such as lowself-esteem, shyness and low assertiveness can predispose people to loneliness andmight also make it more difficult to recover from loneliness $[4,8,11,16,17-18]$.

\section{Limitations of the research}

A convenient sample of size 30 was used. A majority of the sample of age range 19 to 25 years were pursuing a course in Psychology. Hence it was not a representative sample i.e. the sample was homogenous.

\section{Improvements to the research}

Sample size could be increased for more accurate results. A more representative sample could have been used with a wider range in variety in terms of age, education or academia.

Better validated scales could have been used to get more accurate results. Tests like Revised NEO Personality Inventory (NEO PI-I) not only examines the Big Five factors of personality but also its 6 individual facets. The De Jong Gierveld short scales for loneliness could also be substituted for the Revised UCLA Loneliness scale as it examined two levels of this component including emotional and social loneliness. Also, this scale was tested on samples across 7 countries which made it more versatile across cultures.

This is an ongoing research wherein the researchers are still collecting further data from the two different age groups of 19 to 25 years and 40 to 50 years. The aim here is to collect a large sample and conduct linear multiple regression to get predictor values of personality factors to loneliness.

\section{REFERENCES}

1. Perlman D, Peplau LA. Toward a social psychology of loneliness. Personal Relat 1981;3:31-56.

2. Chodorow NJ. The reproduction of mothering: Psychoanalysis and the sociology of gender. Univ of California Press; 1999. 
3. Havens B, Hall M. Social isolation, loneliness, and the health of older adults in Manitoba, Canada. Indian J Gerontol 2001;15(1-2):126-44.

4. Dykstra PA, Van Tilburg TG, Gierveld JD. Changes in older adult loneliness: Results from a seven-year longitudinal study. Res Aging 2005;27(6):725-47.

5. Woodward JC, Zabel J, DeCosta C. Loneliness and divorce. J Divorce 1981;4(1):73-82.

6. McCrae RR, John OP. An introduction to the five- factor model and its applications. J Personality 1992;60(2):175-215.

7. Dykstra PA. Older adult loneliness: myths and realities. Eur J Ageing 2009;6(2):91-101.

8. Johann Georg Zimmermann (1785) Über die Einsamkeit.

9. Jones WH, Carver MD. Adjustment and coping implications of loneliness. Handbook of social and Clinical Psychology 1991;42:682-9.

10. John OP, Srivastava S. The Big Five trait taxonomy: History, measurement, and theoretical perspectives. Handbook of personality: Theory and research. 1999;2(1999):102-38

11. Nicolaisen $\mathrm{M}$, Thorsen $\mathrm{K}$. Loneliness among men and women-a five-year follow-up study. Aging Ment Health 2014;18(2):194-206.

12. Woodward JC, Zabel J, DeCosta C. Loneliness and divorce. J Divorce 1981;4(1):73-82.

13. Peplau LA, Cutrona CE. The revised UCLA Loneliness Scale: Concurrent and discriminant validity evidence. J Personal Soc Psychol 1980;39(3):472-80.

14. Jones WH, Carpenter BN, Quintana D. Personality and interpersonal predictors of loneliness in two cultures. J Personal Soc Psychol 1985;48(6):1503-10.

15. Windle G, Woods RT. Variations in subjective wellbeing: The mediating role of a psychological resource. Ageing Society 2004;24(4):583-602.

16. Dykstra PA, Fokkema T. Social and emotional loneliness among divorced and married men and women: Comparing the deficit and cognitive perspectives. Basic Appl Soc Psychol 2007;29(1):1-2.

17. Vangelisti AL, Perlman D. The Cambridge handbook of personal relationships. Cambridge University Press; 2006.

18. Rokach A, Brock H. The causes of loneliness. Psychology: A Journal of Human Behavior. 1996.

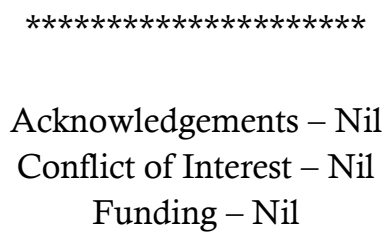

